\title{
CRM-Prozess-Outsourcing in der Telekommunikationsindustrie
}

Die qualitativ-empirische Studie zeigt, ob und welche Customer-Relationship-Management(CRM-)Prozesse bei Telekommunikationsunternehmen ausgelagert werden. Die Entscheidungen für oder gegen ein Outsourcing lassen sich teilweise auf der Basis des Transaktionskostenansatzes begründen. Im Wesentlichen werden nur operative CRM-Prozesse ausgelagert. Dabei sind vor allem Prozesse von niedrigerer Komplexität und strategischer Bedeutung betroffen. Komplexere Prozesse werden nur dann ausgelagert, wenn sie nicht zugleich von hoher strategischer Bedeutung sind. Allerdings liefert dieses Modell nicht für alle Auslagerungsentscheide bezüglich CRM-Prozessen plausible Begründungen, weshalb weitere Motive und Gründe für ein CRMProzess-Outsourcing herangezogen werden.

\section{Inhaltsübersicht}

1 CRM-Prozesse und CRM-ProzessOutsourcing

2 Transaktionskosten als Basis von Outsourcing-Entscheidungen

3 Untersuchung schweizerischer Telekommunikationsunternehmen

3.1 Design der multiplen Fallstudie

3.2 Ausgelagerte CRM-Prozesse

3.3 Motive und Gründe für das CRM-Prozess-Outsourcing

4 Fragen zur Entscheidungsfindung

5 Literatur

\section{CRM-Prozesse und CRM-Prozess- Outsourcing}

Das Customer Relationship Management (CRM) umfasst die kommunikativen Prozesse, mittels der Kunden und Kundenbeziehungen strukturiert bearbeitet werden. Die Abwicklung entsprechender Kommunikationsprozesse erfolgt im Frontoffice. Die Abwicklung von daraus generierten Transaktionsprozessen geschieht im Backoffice. CRM-Prozesse können über unterschiedliche Kommunikationsmedien abgewickelt und vom Kunden oder vom Unternehmen angestoßen werden. CRM-Prozesse lassen sich in langfristig ausgerichtete strategische Kundenbeziehungsprogramme, taktische kundenbeziehungsrelevante Prozesse sowie operative CRM-Prozesse in Form von Marketing-, Verkaufs- und After-Sales-Service-Prozessen differenzieren [Walser 2006].

Eine einheitliche Definition für den Begriff Outsourcing hat sich in der Literatur bislang nicht durchgesetzt. Für diesen Beitrag wird Outsourcing wie folgt definiert: Es handelt sich beim Outsourcing im Kern um die vollständige oder teilweise Auslagerung einmal im eigenen Unternehmen erstellter Leistungen, Funktionen oder Geschäftsprozesse an ein rechtlich und wirtschaftlich unabhängiges Drittunternehmen. Beim Outsourcing von Geschäftsprozessen (Business Process Outsourcing, BPO) geht es um die Vergabe der Durchführung (Management) eines oder mehrerer Geschäftsprozesse an ein externes Drittunternehmen.

Unter CRM-Prozess-Outsourcing kann somit die dauernde oder vorübergehende, partielle oder vollständige Ausgliederung der Abwicklung von Kundenkommunikations- und Beziehungsprozessen sowie entsprechender Supportprozesse verstanden werden.

Im Beitrag soll folgenden Forschungsfragen nachgegangen werden:

1. Welche CRM-Prozesse werden typischerweise ausgelagert? 
2. Weshalb werden diese Prozesse ausgelagert?

3. Welche Einflussgrößen sind beim CRM-Prozess-Outsourcing relevant?

Die erste und zweite Frage lassen sich nicht völlig branchenunabhängig beantworten und werden entsprechend qualitativ-empirisch auf Basis einer multiplen Fallstudie in der schweizerischen Telekommunikationsindustrie (TK-Industrie) untersucht. Die Diskussion der dritten Frage basiert auf einer theoretischen Fundierung, die als Bezugsrahmen für das explorative Vorgehen dient.

\section{Transaktionskosten als Basis von Outsourcing-Entscheidungen}

Zur Erörterung der Zweckmäßigkeit von Outsourcing-Entscheidungen wird sowohl in der englisch- wie in der deutschsprachigen Literatur häufig die Transaktionskostentheorie herangezogen [Picot 1991, S. 340]. Dabei wird eine Transaktion [Picot et al. 1997, S. 66] als Übertragung von Verfügungsrechten definiert. Zentrales Konstrukt ist ein Vertrag. Durch diesen wird die Übertragung geregelt. Transaktionskosten fallen somit im Zusammenhang mit der Gestaltung und Durchsetzung von Verträgen an. [Williamson 1975] unterscheidet mit Bezug zum Vertragsabschluss Ex-ante- und Expost-Transaktionskosten. Eine weitere phasenorientierte Unterscheidung sieht Kosten in der Transaktionsanbahnung, -vereinbarung (ex ante [Bacher 2000, S. 90 f.]), -abwicklung, -kontrolle und -anpassung (ex post) vor [Picot et al. 1985, S. 820]. Die Höhe und Zusammensetzung der Transaktionskosten hängen von den Eigenschaften zu erbringender Leistungen sowie der gewählten Organisationsform ab [Picot 1991, S. 344]. Transaktionskosten können einen Effizienzmaßstab zur Beurteilung und Auswahl institutioneller Koordinations- oder Transaktionsformen [Bacher 2000, S. 87 f.] darstellen. Das Ziel der Transaktionskostenanalyse ist es, für gegebene Transaktionen die effizienteste Orga- nisationsform zu finden [Schätzer 1999, S. 63 f.]. Dabei stellen die »interne Erbringung einer Leistung (Hierarchie) « oder der »externe Bezug der Leistung (Markt)" die beiden Pole eines Kontinuums dar. Ein Outsourcing ist immer dann angebracht, wenn die Transaktionskosten beim Bezug der Leistung von Externen geringer ausfallen. Zentrales Outsourcing-Motiv sind in diesem Theorieansatz (Transaktions-)Kosteneinsparungen.

Zur Erklärung der Zusammenhänge zwischen den Einflussgrößen auf die Transaktionskosten wird das "Organizational failure framework "von Williamson herangezogen [Picot et al. 1997, S. 67; Williamson 1975, S. 40]. Als zentrale Einflussgrößen werden darin die Umweltmerkmale Spezifität, Unsicherheit und Häufigkeit sowie die Verhaltensannahmen Opportunismus und begrenzte Rationalität festgehalten. Die Spezifität (je nach Autor auch die Unsicherheit [Williamson 1975]) wird dabei als ausschlaggebende und die beiden anderen Variablen werden als unterstützende Einflussgrößen genannt. Picot führt als zusätzliche Einflussgröße die strategische Bedeutung an. Für ihn sind strategische Bedeutung und Spezifität die ausschlaggebenden und Unsicherheit und Häufigkeit unterstützende Einflussgrößen. Damit misst Picot der strategischen Bedeutung einen hohen Stellenwert bei. Die Wahl der zwei ausschlaggebenden Einflussgrößen ist wie folgt begründet: Die strategische Bedeutung weist für das Thema dieses Beitrags auf die unternehmensinterne Bedeutung der einzelnen CRM-Prozesse hin. CRM-Prozesse von hoher strategischer Bedeutung sichern einem Unternehmen das Überleben im Wettbewerb. Die Spezifität kann unter Umständen auch ein Hinweis auf die Komplexität (Wissen, Fähigkeiten der Mitarbeiter etc.) eines CRM-Prozesses sein. Die Komplexität ist eher der Einflussgröße Spezifität als der Unsicherheit zuzuordnen. Eine hohe Unsicherheit kann sich aber auch aus einer hohen Komplexität bzw. Spezifität ergeben. 
Aus den Ausprägungen der Einflussgrößen auf die Transaktionskosten lassen sich Normstrategieempfehlungen entwickeln, die im Wesentlichen auf den bei Picot et al. dargestellten Überlegungen zur Portfoliotechnik basieren [Picot et al. 1985, S. 1032 ff.]. Diese sind mit Empfehlungen zu Fremdbezug oder Eigenerstellung in Tabelle 1 aufgeführt. Konzentriert man sich allein auf die zwei zentralen Einflussgrößen strategische Bedeutung und Spezifität, lauten die Empfehlungen wie folgt [Picot et al. 2001, S. 52]: Prozesse von hoher strategischer Bedeutung und hoher Spezifität (Komplexität) gehören zu den Kernkompetenzen und sind selbst zu erbringen ( $\mathrm{N} 1$; $\mathrm{N}$ steht für Normstrategie, die 1 bezeichnet die Normstrategie 1). Prozesse von niedriger strategischer Bedeutung und niedriger Spezifität (Komplexität) sollten dagegen ausgelagert werden (N2). Bei unterschiedlicher Ausprägung der beiden zentralen Einflussgrößen ist eine derart eindeutige Empfehlung nicht mehr möglich. Hier sind die weiteren Einflussgrößen Unsicherheit und Häufigkeit beizuziehen, wodurch sich die Empfehlungen zu den Fällen $\mathrm{N}_{3}$ bis N1o wie in Tabelle 1 dargestellt ergeben.

\section{Untersuchung schweizerischer Telekommunikationsunternehmen}

\subsection{Design der multiplen Fallstudie}

Die Bedeutung und der Entwicklungsstand des CRM sind im Telco-Umfeld hoch. Der intensive Wettbewerb, aber auch die Tatsache, dass es sich um technologieorientierte Dienstleistungen handelt, fördert den Technologieeinsatz im CRM. Zudem sind CRM-Prozessadaptionen aufgrund wechselnder Produkte und Dienstleistungen häufig.

Die Auswahl der Fallstudien ist wie folgt begründbar. Für die Untersuchung wurden alle schweizerischen Mobil- und Festnetzanbieter ohne Internet-Service-Provider ins Auge gefasst. Festnetz- und Mobilkommunikationsanbieter wurden als separate Fälle betrachtet, sofern sie im gleichen Konzern als selbstständige Firmen geführt wurden. Eine Fallübersicht gibt Tabelle 2 wieder.

Auf Basis eines theoriebasierten Interviewleitfadens erfolgte je ein qualitatives Interview im CRM-Kader der sechs untersuchten Unternehmen.

\begin{tabular}{|l|l|l|l|l|l|}
\cline { 2 - 5 } \multicolumn{1}{c|}{} & \multicolumn{4}{c|}{ Ausprägung der Einflussgrößen } & \multicolumn{1}{|l|}{} \\
\hline Normstrategie & Strat. Bedeutung & Spezifität & Unsicherheit & Häufigkeit & Empfehlung \\
\hline N1 & Hoch & Hoch & & & Eigenerstellung \\
\hline N2 & Niedrig & Niedrig & & & Outsourcing \\
\hline N3 & Niedrig & Hoch & Hoch & Hoch & Eigenerstellung \\
\hline N4 & Niedrig & Hoch & Niedrig & Niedrig & Outsourcing \\
\hline N5 & Niedrig & Hoch & Hoch & Niedrig & Outsourcing \\
\hline N6 & Niedrig & Hoch & Niedrig & Hoch & Outsourcing \\
\hline N7 & Hoch & Niedrig & Hoch & Hoch & Eigenerstellung \\
\hline N8 & Hoch & Niedrig & Niedrig & Niedrig & Outsourcing \\
\hline N9 & Hoch & Niedrig & Hoch & Niedrig & Eigenerstellung \\
\hline N10 & Hoch & Niedrig & Niedrig & Hoch & Eigenerstellung \\
\hline
\end{tabular}

Tab. 1: Ableitung transaktionskostentheoretischer Normstrategien [Picot 1991, S. 349 ff.; Picot et al. 1997, S. 70; Bacher 2000, S. $100 \mathrm{ff}$.] 


\begin{tabular}{|l|l|l|l|}
\hline Fall & Angebot & Strategieart & $\begin{array}{l}\text { Tendenzen bezüglich CRM-Prozess- } \\
\text { Outsourcing }\end{array}$ \\
\hline 1 & $\begin{array}{l}\text { Festnetz- \& } \\
\text { Internet-Provider }\end{array}$ & $\begin{array}{l}\text { Differenzierungsstrategie } \\
\text { durch Service (After Sales } \\
\text { Service) sowie wenn mög- } \\
\text { lich durch Produktführer- } \\
\text { schaft }\end{array}$ & $\begin{array}{l}\text { Eigenerstellung strategischer und mehrheitlich } \\
\text { taktischer Prozesse, Eigenerstellung After- } \\
\text { Sales-Service-Prozesse auf operativer Ebene } \\
\text { auch wegen gut integrierter Front- und Back- } \\
\text { office-Systeme }\end{array}$ \\
\hline 2 & $\begin{array}{l}\text { Kabelnetz- \& } \\
\text { Internet-Provider }\end{array}$ & $\begin{array}{l}\text { Bisher Produktdifferenzie- } \\
\text { rungsstrategie, Wechsel } \\
\text { aufgrund von Marktentwick- } \\
\text { lungsgegebenheiten möglich }\end{array}$ & $\begin{array}{l}\text { Eigenerstellung strategischer und taktischer } \\
\text { CRM-Prozesse, tendenziell Eigenerstellung von } \\
\text { Marketing und After Sales Service oder } \\
\text { Customer Care }\end{array}$ \\
\hline 3 & $\begin{array}{l}\text { Mobilkommunika- } \\
\text { tions-Provider }\end{array}$ & $\begin{array}{l}\text { Differenzierung über Ser- } \\
\text { vice, Produktdifferenzierung } \\
\text { wird als schwierig erachtet }\end{array}$ & $\begin{array}{l}\text { Eigenerstellung strategischer und mehrheitlich } \\
\text { taktischer Prozesse }\end{array}$ \\
\hline 4 & $\begin{array}{l}\text { Mobilkommunika- } \\
\text { tions-Provider }\end{array}$ & $\begin{array}{l}\text { Differenzierung durch Quali- } \\
\text { tät, Qualitätsführerschaft }\end{array}$ & $\begin{array}{l}\text { Eigenerstellung strategischer und taktischer } \\
\text { Prozesse, tendenziell Verkaufsprozess- } \\
\text { Outsourcing, Marketing- und After-Sales- } \\
\text { Service-Prozesse tendenziell inhouse }\end{array}$ \\
\hline 5 & $\begin{array}{l}\text { Vollservice- } \\
\text { Provider }\end{array}$ & $\begin{array}{l}\text { Differenzierungsstrategie } \\
\text { relativ zu Produkten und } \\
\text { Kundensegmenten }\end{array}$ & $\begin{array}{l}\text { Gewisse Marketing- und Verkaufsprozesse } \\
\text { werden an Outsourcer vergeben }\end{array}$ \\
Dienstleistungen
\end{tabular}

Tab. 2: Für das CRM relevante unternehmensstrategische Schwerpunkte der untersuchten Unternehmen

\subsection{Ausgelagerte CRM-Prozesse}

Die unternehmensstrategischen Schwerpunkte der befragten Unternehmen können aufgrund der Aussagen der Interviewpartner wie in Tabelle 2 charakterisiert werden. Die Unternehmen werden zur Darstellung der empirischen Resultate in den folgenden Tabellen mit Nummern bezeichnet, da die Firmennamen aus Geheimhaltungsgründen nicht publiziert werden dürfen.

Eine tabellarische Darstellung der langfristig ausgelegten strategischen CRM-Programme zur Kundengewinnung, zum Cross- und Up-Selling, zur Kundenbindung sowie zur Kundenrückgewinnung erübrigt sich, weil hier überall Eigenerstellung angegeben wurde. Aus transaktionskostentheoretischer Perspektive weisen diese Prozesse im Allgemeinen eine hohe strategische Bedeutung und Spezifität auf. Die in der Untersuchung erzielten Resultate bestäti- gen die in $\mathrm{N} 1$ abgeleitete Empfehlung hinsichtlich der Make-or-Buy-Entscheidung.

Nachfolgend werden überwiegend tabellarisch für verschiedene CRM-Prozesse die Ausprägungen bezüglich Eigenerstellung $(\mathrm{E})$ und Outsourcing $(\mathrm{O})$ bei den jeweiligen Unternehmen wiedergegeben, sofern in den Interviews konkrete Auskünfte dazu erhältlich waren (vgl. Tab. 3). Der Vermerk Eigenerstellung (E) bedeutet nicht notwendigerweise, dass die Leistung nur selbst erstellt wird. Es sind hier auf einem Kontinuum von nur selbst erstellt bis teilweise selbst erstellt verschiedene Ausprägungen möglich. Ebenfalls bedeutet der Vermerk Outsourcing (O) nicht einfach ein vollständiges Outsourcing. Es kann sich dabei auch um partielle oder temporäre Outsourcing-Lösungen mit einem oder verschiedenen Partnern handeln. Insofern sind die Angaben in der Tabelle vereinfacht und mit der nötigen Vorsicht zu 
interpretieren. Wenn keine oder widersprüchliche Auskünfte vorlagen, wurde ein Strich gesetzt.

Bei der Darstellung zu den taktischen CRMProzessen in Tabelle 3 und der Darstellung zu den operativen CRM-Prozessen in Tabelle 4 ergibt sich bei den jeweiligen Unternehmen ein gemischtes Bild. Bei der vergleichenden Beurteilung der Ausprägungen ergeben sich an verschiedenen Orten Übereinstimmungen zwischen Unternehmensstrategie und Outsourcing-Strategien. Bemerkenswert sind etwa sehr differierende Ausprägungen des Make-or-Buy von CRM-Prozessen in den Fallstudien bei Customer-Care- oder After-Sales-Service-Prozessen. Aus Sicht der Transaktionskostentheorie weisen diese Prozesse (bis auf zwei Ausnahmen) entweder eine hohe strategische Bedeutung oder eine hohe Spezifität auf. Die in N3 bis N1o abgeleiteten Empfehlungen hinsichtlich einer Make-or-Buy-Entscheidung werden von den erzielten Resultaten nicht bestätigt. Dies weist darauf hin, dass der auf Transaktionskosten basierende Erklärungsansatz nicht für alle CRMProzess-Outsourcing-Sachverhalte dienlich ist. Beim Entscheid für ein Outsourcing ist, wie Abschnitt 3.3 zeigt, von verschiedenen Gründen und Motiven auszugehen, etwa dem Kundenwert oder dem Kapazitätsmanagement.

Interessant ist nicht nur, ob und bei welchen CRM-Prozessen ein Outsourcing stattfindet, sondern auch, mit wie vielen externen Partnern dabei gearbeitet wird. Tabelle 5 zeigt von den Interviewpartnern genannte Arten und Anzahlen von Outsourcing-Partnern.

\subsection{Motive und Gründe für das CRM-Prozess-Outsourcing}

Die meistgenannten Motive und Gründe für ein CRM-Prozess-Outsourcing lauten bezüglich der nachfolgend näher beschriebenen Items wie folgt (in Klammern ist die Anzahl Nennungen (mit möglichen Mehrfachnennungen) bei sechs befragten Unternehmen angegeben): Kosten (6), Kapazitätsüberlastung (4), Qualität (4), Konzentration auf das Kerngeschäft (3), Mangel an Know-how (3) und Flexibilität (2). Hinsichtlich ihrer Bedeutung unterscheiden sich die genannten Motive und Gründe je nach Outsourcing-Situation bzw. -Strategie. So lassen sich vier Outsourcing-Situationen bzw. -Strategien unterscheiden: Kapazitätsüberlastung (Peak- und Overflow-Management), mangelnde Fähigkeiten und Know-how, Konzentration

\begin{tabular}{|c|c|c|c|c|c|c|c|}
\hline \multirow[t]{2}{*}{ CRM-Prozesskategorie } & \multirow[t]{2}{*}{ Taktische CRM-Prozesse } & \multicolumn{6}{|c|}{ Ausprägung in Fallstudien } \\
\hline & & 1 & 2 & 3 & 4 & 5 & 6 \\
\hline \multirow{4}{*}{$\begin{array}{l}\text { Komplexe analytische } \\
\text { CRM-Prozesse }\end{array}$} & Data-Mining & $\mathrm{E}$ & $\mathrm{E}$ & $\mathrm{E}$ & $\mathrm{E}$ & $E$ & - \\
\hline & OLAP & $\mathrm{E}$ & $E$ & $\mathrm{E}$ & $\mathrm{E}$ & $E$ & - \\
\hline & Kundenverhaltensmodellierung & $\mathrm{E}$ & $\mathrm{E}$ & $\mathrm{E}$ & $\mathrm{E}$ & $E$ & - \\
\hline & Kunden-Scoring-Modellierung & $E$ & $E$ & $E$ & $\mathrm{E}$ & $E$ & - \\
\hline \multirow{4}{*}{$\begin{array}{l}\text { Standardisierbare analytische } \\
\text { CRM-Prozesse }\end{array}$} & Internetportal-Reporting & $\mathrm{O}$ & $E$ & $\mathrm{E}$ & $\mathrm{E}$ & $E$ & - \\
\hline & Click-Streams & $\mathrm{O}$ & $\mathrm{E}$ & $\mathrm{E}$ & $\mathrm{E}$ & $E$ & - \\
\hline & Datenintegration handgeschriebener Daten & - & - & $\mathrm{O}$ & 0 & $\mathrm{O}$ & - \\
\hline & Adress(listen)-Management & 0 & - & - & 0 & - & - \\
\hline \multirow{2}{*}{$\begin{array}{l}\text { Komplexe taktische } \\
\text { CRM-Prozesse }\end{array}$} & Planung neuartiger Marketingkampagnen & $\mathrm{E}$ & $\mathrm{E}$ & $E$ & $\mathrm{E}$ & $\mathrm{E}$ & - \\
\hline & Multi-Channel-Kampagnen & $E$ & $\mathrm{E}$ & $\mathrm{E}$ & $\mathrm{E}$ & $E$ & - \\
\hline \multirow{2}{*}{$\begin{array}{l}\text { Standardisierbare taktische } \\
\text { CRM-Prozesse }\end{array}$} & Planung von Promotionsaktionen & $\mathrm{E}$ & $\mathrm{E}$ & $\mathrm{E}$ & $\mathrm{E}$ & $E$ & - \\
\hline & Planung von Doorknockers-Aktionen & - & $E$ & $\mathrm{E}$ & $\mathrm{E}$ & $\mathrm{E}$ & - \\
\hline
\end{tabular}

Tab. 3: Outsourcing taktischer CRM-Prozesse - qualitativ-empirische Resultate 


\begin{tabular}{|c|c|c|c|c|c|c|c|}
\hline \multirow[t]{2}{*}{ CRM-Prozesskategorie } & \multirow[t]{2}{*}{ Operative CRM-Prozesse } & \multicolumn{6}{|c|}{ Ausprägung in Fallstudien } \\
\hline & & 1 & 2 & 3 & 4 & 5 & 6 \\
\hline \multirow[t]{9}{*}{ Marketingprozesse } & Durchführung neuartiger Kampagnen & $\mathrm{E}$ & $\mathrm{E}$ & $\mathrm{E}$ & $\mathrm{E}$ & $\mathrm{E}$ & - \\
\hline & Durchführung Multi-Channel-Kampagnen & $\mathrm{E}$ & $\mathrm{E}$ & $\mathrm{E}$ & $\mathrm{E}$ & $E$ & - \\
\hline & Durchführung Direct-Mail-Kampagnen & $\mathrm{O}$ & $\mathrm{E}$ & $E$ & $\mathrm{E}$ & $E$ & $\mathrm{O}$ \\
\hline & Durchführung Direct-Email-Kampagnen & 0 & $E$ & $\mathrm{E}$ & $\mathrm{E}$ & $\mathrm{E}$ & 0 \\
\hline & Telemarketing & $\mathrm{O}$ & $\mathrm{E}$ & 0 & $\mathrm{O}$ & $\mathrm{O}$ & $\mathrm{O}$ \\
\hline & Promotionsaktionen & - & - & $\mathrm{O}$ & - & $\mathrm{O}$ & $\mathrm{O}$ \\
\hline & Doorknocker-Aktionen & - & $\mathrm{O}$ & - & - & - & $\mathrm{O}$ \\
\hline & Durchführung von SMS-Kampagnen & - & - & $E$ & - & - & - \\
\hline & Kundenbefragungen & - & - & - & - & $\mathrm{O}$ & - \\
\hline \multirow[t]{4}{*}{ Verkaufsprozesse } & Fachhandel & - & $\mathrm{O}$ & $\mathrm{O}$ & 0 & 0 & $\mathrm{O}$ \\
\hline & Telesales & $\mathrm{O}$ & $\mathrm{O}$ & $\mathrm{O}$ & - & 0 & $\mathrm{O}$ \\
\hline & Durchführung des Cross-Sellings & $\mathrm{O}$ & $\mathrm{O}$ & $E$ & 0 & 0 & $\mathrm{O}$ \\
\hline & Durchführung des Up-Sellings & $\mathrm{O}$ & $\mathrm{O}$ & $\mathrm{E}$ & $\mathrm{O}$ & $\mathrm{O}$ & 0 \\
\hline \multirow{10}{*}{$\begin{array}{l}\text { Customer-Care- oder } \\
\text { After-Sales-Service- } \\
\text { Prozesse }\end{array}$} & Produktregistration & $E$ & $E$ & $\mathrm{O}$ & $E$ & $E$ & $\mathrm{O}$ \\
\hline & Produktinformation & $E$ & $\mathrm{E}$ & 0 & $E$ & $E$ & $\mathrm{O}$ \\
\hline & Preisanfragen & $\mathrm{E}$ & $E$ & 0 & $\mathrm{E}$ & $E$ & 0 \\
\hline & Rechnungsanfragen & $E$ & $\bar{E}$ & $\mathrm{O}$ & $\mathrm{E}$ & $E$ & $\mathrm{O}$ \\
\hline & Adressänderungen & $\mathrm{E}$ & $\mathrm{E}$ & 0 & $\mathrm{E}$ & $\mathrm{E}$ & $\mathrm{O}$ \\
\hline & Standardisierbare Beschwerdeprozesse & $E$ & $\mathrm{E}$ & 0 & $E$ & $E$ & $\mathrm{O}$ \\
\hline & Komplexe Beschwerdeprozesse & $\mathrm{E}$ & $E$ & $E$ & $\mathrm{E}$ & $\mathrm{E}$ & $\mathrm{O}$ \\
\hline & Standardisierbare techn. Supportprozesse & $\mathrm{O}$ & $E$ & 0 & $E$ & 0 & $\mathrm{O}$ \\
\hline & Komplexe technische Supportprozesse & $E$ & $\mathrm{E}$ & 0 & $\mathrm{E}$ & $\mathrm{E}$ & $\mathrm{O}$ \\
\hline & Sprachauskunftsdienst & - & 0 & 0 & $\mathrm{E}$ & $\mathrm{O}$ & $\mathrm{O}$ \\
\hline \multirow[t]{6}{*}{ Administrative Prozesse } & Rückerstattungen & $E$ & $\mathrm{E}$ & $E$ & $E$ & $E$ & - \\
\hline & Betreibungen & $E$ & $\mathrm{E}$ & $\mathrm{E}$ & $E$ & $E$ & - \\
\hline & Druck von Rechnungen & - & 0 & 0 & - & - & $\mathrm{O}$ \\
\hline & Versand von Produkten & - & 0 & - & - & - & $\mathrm{O}$ \\
\hline & Versand von Rechnungen & - & - & 0 & - & - & $\mathrm{O}$ \\
\hline & Druck von Rechnungsbeilagen & - & - & 0 & - & - & 0 \\
\hline
\end{tabular}

Tab. 4: Outsourcing operativer CRM-Prozesse - qualitativ-empirische Resultate

auf das Kerngeschäft sowie Kostenvariabilisierung. Je nach Outsourcing-Situation bzw. -Strategie werden CRM-Prozesse in unterschiedlichem Maße ausgelagert.

\section{Strategische Motive und Gründe}

Konzentration auf das Kerngeschäft: Im Rahmen dieser Outsourcing-Strategie werden CRM-Pro- zesse von geringer strategischer Bedeutung und Komplexität ausgelagert. Es weisen entsprechende externalisierte CRM-Prozesse weder für Kunden noch das eigene Unternehmen einen Mehrwert auf. Die Wertschöpfung ist gering. Durch eine Auslagerung derartiger CRMProzesse werden Ressourcen freigesetzt, die für das Kerngeschäft eingesetzt werden können. 


\begin{tabular}{|c|c|c|}
\hline Fall & Genutzte Outsourcing-Partner & $\begin{array}{l}\text { Anzahl Outsourcing-Partner je } \\
\text { CRM-Prozessbereich }\end{array}$ \\
\hline 1 & $\begin{array}{l}\text { Direct-Marketing-Agenturen, Kreativ-Agenturen, Direct- } \\
\text { Marketing-Center, weitere externe Callcenter für Telesales und } \\
\text {-Marketing, Portal-Reporting-Partner, Adresslieferant }\end{array}$ & $\begin{array}{l}\text { Es waren keine Anzahlen } \\
\text { erhältlich }\end{array}$ \\
\hline 2 & Kundenkommunikation; Building-Upgrade und Field-Service & $\begin{array}{l}\text { 4-5 Outsourcing-Partner sowie } \\
\text { mehrere Hundert Partner für } \\
\text { Teilbereiche }\end{array}$ \\
\hline 3 & $\begin{array}{l}\text { Anbieter für Customer-Care-Prozesse; Anbieter für Outbound- } \\
\text { Kampagnen sowie Anbieter im Bereich indirekter Verkauf }\end{array}$ & $\begin{array}{l}4 \text { Partner für Customer Care/After } \\
\text { Sales Service; 4-6 Outbound- } \\
\text { Partner; diverse Partner im } \\
\text { Verkauf }\end{array}$ \\
\hline 4 & Keine Angaben & Keine Angaben \\
\hline 5 & $\begin{array}{l}\text { Callcenterpartner, Verkaufspartner Telesales, Partnerschaften mit } \\
\text { Fachhandel und für Durchführung externalisierter Fulfillment- und } \\
\text { Backoffice-Prozesse }\end{array}$ & $\begin{array}{l}\text { Es waren keine Anzahlen } \\
\text { erhältlich }\end{array}$ \\
\hline 6 & $\begin{array}{l}\text { Partnerschaften mit diversen Callcentern sowie Fulfillment- und } \\
\text { Backoffice-Prozesspartner }\end{array}$ & $\begin{array}{l}\text { Es waren keine Anzahlen } \\
\text { erhältlich }\end{array}$ \\
\hline
\end{tabular}

Tab. 5: Art und Anzahl genutzter Outsourcing-Partner

Ferner lassen sich hier durch ein Outsourcing auch Kosten senken. Administrative Prozesse bzw. Backoffice- und Fulfillment-Prozesse werden im Rahmen dieser Outsourcing-Strategie ausgelagert.

\section{Kostenreduktion und -variabilisierung:}

Durch eine Auslagerung von CRM-Prozessen im Rahmen dieser Outsourcing-Strategie können Kosten eingespart und/oder variabilisiert werden. Dadurch wird ein Aufbau unnötiger Ressourcen und Kapazitäten vermieden. Insbesondere die Durchführung verschiedener Marketingkampagnen wird im Rahmen dieser Strategie externalisiert.

\section{Operative Motive und Gründe}

Kapazitätsüberlastung (Peak- und Overflow-Management): Ein Outsourcing im Rahmen eines Overflow-Managements bezüglich CRM-Prozessen beschreibt die Situation der Kapazitätsüberlastung, in der Prozesse ausgelagert werden, die von unternehmensinternen Kapazitäten nicht absorbiert werden können. Dadurch wird laufender Kapazitätsausgleich möglich. Durch den Aufbau zusätzlicher unternehmens- interner Kapazitäten könnte eine Kapazitätsüberlastung vermieden werden. Diese zusätzlich aufgebauten Kapazitäten werden später eventuell nicht mehr benötigt, gehen dann jedoch in Folge der Kostenremanenz immer noch zulasten des Unternehmens. Durch die Auslagerung von CRM-Prozessen im Rahmen eines OverflowManagements kann sich ein Unternehmen einen Flexibilitätsspielraum bezüglich Kapazitätsproblemen und Kostenreduktionen schaffen. Ähnliches gilt für die Auslagerung von CRMProzessen im Rahmen eines Peak-Managements. Oft führen kurz- bis mittelfristige Marketingaktivitäten zu Kapazitätsüberlastungen infolge zeitweiliger Spitzenbedarfe. Im Gegensatz zum Overflow-Management ist die Kapazitätsüberlastung beim Peak-Management eher kurzfristiger Natur. Im Normalfall werden die ausgelagerten CRM-Prozesse, sobald die Belastungsspitze vorüber ist, wieder vollständig vom eigenen Unternehmen abgewickelt. Technische Supportprozesse, Beschwerde-, Informationsund Anschlussaktivierungsprozesse werden im Rahmen des Peak- und Overflow-Managements als Outsourcing-Kandidaten genannt. 
Mangelnde Fähigkeiten und Know-how: Ein Outsourcing von CRM-Prozessen aufgrund mangelnder Fähigkeiten oder mangelnden Know-hows des Personals beschreibt eine Situation, in der für die Abwicklung von CRMProzessen unternehmensintern Wissenslücken vorhanden sind oder eigene Mitarbeiter nicht über benötigte Fähigkeiten für die Durchführung bestimmter CRM-Prozesse verfügen. Durch eine Auslagerung kann einerseits externes Wissen eingekauft werden. Andererseits kann dadurch eine Qualitätsverbesserung bezüglich der ausgelagerten CRM-Prozesse durch das Outsourcing erreicht werden. Sofern das benötigte Wissen unternehmensintern absorbiert werden kann, werden ausgelagerte CRMProzesse wieder ins eigene Unternehmen integriert. Am Markt gibt es Unternehmen, die sich auf bestimmte Bereiche spezialisiert haben, etwa auf Direktmarketing, Promotionsaktionen oder Telemarketing. Aufgrund ihrer Spezialisierung erzielen diese Unternehmen bei der Durchführung bestimmter CRM-Prozesse höhere Qualitäten und Deckungsbeiträge, als dies unternehmensintern möglich wäre. Im Allgemeinen werden aufgrund eines Mangels an Know-how oder entsprechender Mitarbeiterfähigkeiten (die Durchführung) eher komplexere( $r$ ) Prozesse ausgelagert, etwa Cross- und Up-Selling-Prozesse sowie technische Supportprozesse (in einem Beispiel z.B. für Blackberrys; dabei geht hier der Support mehr in Richtung IT-Support, was wiederum nicht zu den Kernkompetenzen des entsprechenden TK-Unternehmens gehört).

\section{Zusammenfassung empirischer Ausprägungen von Motiven und Gründen}

In Tabelle 6 werden die empirischen Ausprägungen von strategischen und operativen Motiven und Gründen des CRM-Prozess-Outsourcings über die sechs untersuchten Unternehmen zusammengefasst. Für das Unternehmen 3 gilt in Ergänzung zu dem, was in Tabelle 6 angemerkt ist: Ausgehend von Kunden,
Produkten und Komplexität erfolgt eine Erstellung von Prozess-Outsourcing-Landkarten, auf deren Basis situative oder definitive, temporäre oder dauerhafte Outsourcing-Entscheidungen getroffen werden.

\section{Fragen zur Entscheidungsfindung}

Ausgehend von den in diesem Beitrag gemachten Äußerungen stellt sich die Frage nach einer systematischen Erfassung von Entscheidungen für oder wider ein CRM-Prozess-Outsourcing. Dies kann in Form von Fragen erfolgen, die in vier Gruppen aufgeteilt werden.

- Unternehmensstrategie: Vertritt das Unternehmen eine Differenzierungsstrategie oder eine Kostenführerschaftsstrategie? Differenziert sich das Unternehmen bezüglich der Kundenbeziehung oder bezüglich des Leistungsangebots? Wenn die Kundenbeziehung der Differenzierungsfaktor ist, erfolgt eine Differenzierung bezüglich strategischer (Gewichtung der CRM-Programme in Relation zueinander) oder operativer CRM-Prozesse (stärkere Fokussierung auf Marketing, Verkauf oder After Sales Service)?

- Outsourcing-Strategie bezüglich CRM-Prozessen: Um welche CRM-Prozesskategorie handelt es sich: strategische, taktische oder operative CRM-Prozesse? Welche Ausprägungen haben diese Prozesse bezüglich strategischer Bedeutung, bezüglich Spezifität und Komplexität, aber auch bezüglich Unsicherheit und Häufigkeit des Anfalls entsprechender Prozesse? Was ergeben sich daraus für Normstrategien?

- Motive und Gründe auf operativer Ebene: Wie sieht die Kapazitätssituation an den Kundenkontaktpunkten aus (Filiale, Kontaktcenter, Web etc.)? Welche Flexibilität fordert oder wünscht das Unternehmen in der operativen CRM-Prozessabwicklung? Welches Knowhow bezüglich Kunden und Leistungsangebot sowie welche Komplexität bezüglich Kundenbeziehungen und Leistungsangebot ist 


\begin{tabular}{|c|c|c|}
\hline Fall & Motive & Gründe \\
\hline \multirow[t]{2}{*}{1} & Kosten und Qualität & $\begin{array}{l}\text { Zusammenarbeit mit Partnern und deren Skills erlauben } \\
\text { Qualitätssteigerung der Prozesse und Ersatz fixer durch } \\
\text { variable Kosten }\end{array}$ \\
\hline & $\begin{array}{l}\text { Outsourcing technischer Supportprozesse } \\
\text { bei der Überlastung von Kapazitäten }\end{array}$ & $\begin{array}{l}\text { Overflow-Management, da Eigenaufbau von Kapazitäten } \\
\text { dafür zu teuer }\end{array}$ \\
\hline \multirow[t]{4}{*}{2} & $\begin{array}{l}\text { Flexibilität, Skalierbarkeit und durch } \\
\text { Outsourcing resultierende } \\
\text { Kosteneinsparungen }\end{array}$ & $\begin{array}{l}\text { Outsourcing ermöglicht freiere Gestaltung der } \\
\text { Kapazitätsbereitstellungen bei gleichzeitiger Bereithaltung } \\
\text { externer Ressourcen (sofern Know-how vorhanden) }\end{array}$ \\
\hline & Probleme im operativen Bereich & $\begin{array}{l}\text { Rasche Entwicklung des Providers erforderte zunächst } \\
\text { technische Investitionen, Qualität und Kapazitäten der } \\
\text { CRM-Prozesse mussten mit Outsourcing erhöht werden }\end{array}$ \\
\hline & $\begin{array}{l}\text { Zunehmendes Insourcing (etwa von } \\
\text { Telesales-Prozessen) }\end{array}$ & $\begin{array}{l}\text { Aufgrund zunehmender Beherrschung Prozessprobleme } \\
\text { und Rebalancing der Investitionen }\end{array}$ \\
\hline & Konzentration auf Kerngeschäft & $\begin{array}{l}\text { Auslagerung von Prozessen, die keinen Mehrwert für } \\
\text { Unternehmen generieren (z.B. administrative Prozesse) }\end{array}$ \\
\hline \multirow[t]{4}{*}{3} & $\begin{array}{l}\text { Outsourcing-Unterscheidung bezüglich der } \\
\text { folgenden Objekte: Produkte, } \\
\text { Kunden(segmente); in Relation dazu } \\
\text { stehen Komplexität, Infrastruktur, } \\
\text { Flexibilitätsbedarf (Kapazitätsüberlastung), } \\
\text { Kosten und Qualität als weitere Motive }\end{array}$ & $\begin{array}{l}\text { Aufgrund von Bedarf an Flexibilität, Kosten und Qualität in } \\
\text { Relation zu Produkten und Kundensegmenten situativ sich } \\
\text { ergebende und sich verändernde Outsourcing-Situation }\end{array}$ \\
\hline & Vorhandene (integrierte) Infrastruktur & $\begin{array}{l}\text { Aufbau Cross- und Up-Selling-Callcenter und eigener } \\
\text { SMS-Kampagnen-Infrastruktur machen Outsourcing wenig } \\
\text { sinnvoll }\end{array}$ \\
\hline & Qualität & $\begin{array}{l}\text { Im Markt qualitativ bessere Fähigkeiten vorhanden als } \\
\text { inhouse }\end{array}$ \\
\hline & Technisches Know-how & $\begin{array}{l}\text { Beispiel: Outsourcing Blackberrys-Technologie-Support; } \\
\text { entspricht eher IT-Support als Telco-Tech-Support: eigener } \\
\text { Know-how-Aufbau zu teuer }\end{array}$ \\
\hline \multirow[t]{4}{*}{4} & Branchenüblichkeit & Auslagerung von Verkaufsprozessen an Fachhandel \\
\hline & Kapazitätsüberlastungen & Peak- und Overflow-Management \\
\hline & $\begin{array}{l}\text { Wertschöpfungsbeitrag (strategische } \\
\text { Bedeutung) }\end{array}$ & $\begin{array}{l}\text { Administrationsprozesse (geringe strategische } \\
\text { Bedeutung), die einen geringen Wertschöpfungsbeitrag } \\
\text { leisten und nicht zur Differenzierung beitragen }\end{array}$ \\
\hline & Kosten (u.a. versus Know-how) & $\begin{array}{l}\text { Durch Outsourcing Kostensenkung oder } \\
\text { Kostenvariabilisierung, aber auch Kostenreduktion durch } \\
\text { Nichtaufbau von Know-how }\end{array}$ \\
\hline \multirow[t]{2}{*}{5} & $\begin{array}{l}\text { Kapazitätsüberlastung relativ zu } \\
\text { Kostenreduktion (Outsourcing billiger als } \\
\text { Eigenerstellung) }\end{array}$ & $\begin{array}{l}\text { Kundenzufriedenheitsbefragungen und Durchführung von } \\
\text { Kundengewinnungsaktionen }\end{array}$ \\
\hline & Know-how-Mangel und Qualitätsprobleme & $\begin{array}{l}\text { Mitarbeiter verfügen nicht über Know-how und } \\
\text { Outsourcing-Partner liefert (bessere oder gute) } \\
\text { Qualitätsprozesse }\end{array}$ \\
\hline 6 & $\begin{array}{l}\text { Kostensenkung und Vermeidung } \\
\text { Kapazitätsaufbau (Lean-Management) }\end{array}$ & Kostenführerschaftsstrategie \\
\hline
\end{tabular}

Tab. 6: Motive und Gründe für das CRM-Prozess-Outsourcing 
an den Kundenkontaktpunkten bestimmend für den Erfolg? Welche Qualitätsüberlegungen sind hinsichtlich der Kundenbeziehungsprozesse und des Leistungsportfolios für den Erfolg erforderlich?

- Zeitliche und kostenspezifische Ausprägungen des CRM-Prozess-Outsourcings: Handelt es sich um eine temporäre oder dauerhafte Ausgliederung der CRM-Prozesse? Handelt es sich um eine vollständige oder teilweise Ausgliederung der CRM-Prozesse? Wird tendenziell auf eine Wiedereingliederung oder auf eine immer größere Ausgliederung des oder der CRM-Prozesse hin gearbeitet?

Ausgehend von den hier gemachten Aussagen zum CRM-Prozess-Outsourcing ist in der künftigen Forschung vertieft der Frage nachzugehen, welche theoretischen Konstrukte neben der Transaktionskostentheorie zur Erklärung des Outsourcing-Verhaltens im CRM-Prozessbereich herangezogen werden können. Zudem sollten mit dem gleichen Forschungsdesign auch andere Branchen hinsichtlich der Outsourcing-Potenziale im CRM-Prozessbereich untersucht werden, um die allgemeinen Aussagen breiter fundieren zu können.

\section{Literatur}

[Bacher 2000] Bacher, M. R.: Outsourcing als strategische Marketing-Entscheidung. DUV, Wiesbaden, 2000.

[Picot 1991] Picot, A.: Ein neuer Ansatz zur Gestaltung der Leistungstiefe. In: Zeitschrift für betriebswirtschaftliche Forschung, 43. Jg., 1991, Heft 4, S. 336-357.

[Picot et al. 1985] Picot, A.; Reichwald, H.; Schönekker, G.: Eigenerstellung oder Fremdbezug von Organisationsleistung - Ein Problem der Unternehmensführung (I). In: Office Management, 33. Jg., 1985, Heft 9, S. 818-821.

[Picot et al. 1997] Picot, A.; Dietl, H.; Franck, E.: Organisation - Eine ökonomische Perspektive. Schäffer-Poeschel, Stuttgart, 1997.
[Picot et al. 2001] Picot, A.; Reichwald, R.; Wigand, $R$. $T$.: Die grenzenlose Unternehmung - Information, Organisation und Management. Gabler, Wiesbaden, 2001.

[Schätzer 1999] Schätzer, S.: Unternehmerische Outsourcing-Entscheidungen: Eine transaktionskostentheoretische Analyse. DUV, Wiesbaden, 1999.

[Walser 2006] Walser, K.: Auswirkungen des CRM auf die IT-Integration. Eul Verlag, Lohmar bei Köln, 2006.

[Williamson 1975] Williamson, O. E.: Markets and Hierarchies: Analysis and Antitrust Implications. A Study in the Economies of Internal Organization. The Free Press, New York, 1975.

Dr. rer. oec. Konrad Walser

Senior Researcher

Berner Fachhochschule

Kompetenzzentrum für Public

Management und E-Government

Morgartenstr. 2a/Postfach 305

$\mathrm{CH}-3000$ Bern 22

konrad.walser@bfh.ch

www.wirtschaft.bfh.ch

Prof. Dr. Thomas Myrach

Universität Bern

Institut für Wirtschaftsinformatik

Abteilung Information Management

Engehaldenstr. 8

$\mathrm{CH}-3012$ Bern

thomas.myrach@iwi.unibe.ch

www.im.iwi.unibe.ch

lic. rer. pol. Marin Gimpert

Expert/Evaluator

BNS-Group AG

Bahnhofstr. 50

$\mathrm{CH}-2502$ Biel

marin.gimpert@bns-group.com

www.bns-group.com 